\title{
ABDUCTIVE REASONING AS THE LOGIC OF AGENT-BASED MODELLING
}

\author{
Matus Halas \\ Department of International Relations \\ Charles University \\ U krize 8, 15800 Prague, Czech Republic \\ E-mail: matus.halas@gmail.com
}

\section{KEYWORDS}

Abduction, pragmatism, exploration, research design.

\begin{abstract}
This paper is focused on methodological problems implicit in agent-based modelling of the complex social systems, and in the related multi-agent computer simulations that are widely used to generate necessary quantitative data. After describing the consequences of (non)linear relations and complexity for social science research, I proceed to cope with abductive reasoning as an alternative way of proposing new hypotheses. Finally, I try to turn this into a general framework for any research design based on agent-based modelling and multi-agent simulations within social sciences.
\end{abstract}

\section{INTRODUCTION}

To begin with, one must first clearly distinguish complex systems, agent-based models, and multi-agent simulations. Differences between them are not settled even in the scholarly literature and you can easily find expressions like complexity theory, agent-based simulations, or multi-agent systems. But in this text I will use complex systems only as a label for real world phenomena, agent-based models as a way of their theoretical simplification, and multi-agent simulations as a method of data acquisition. There are of course other possible methods, but multi-agent simulations are the best way how to put into praxis agent-based models of complex systems. These models and related multiagent computer simulations are at the same time widely used in natural as well as social sciences.

Qualitative methods and case studies approach are generally considered as being well suited for explaining complex phenomena and providing the whole and satisfactory picture of various problems in social sciences. That is because in such research design one often looks at only very few cases with lots of considered variables (Bennett and Elman 2007: 171). On the other hand, quantitative methods try to explain as many cases as possible with help of the fewest possible assumptions, while at the same time attempting to identify key variables behind the linear relationship of explanans and explanandum.

However, a special kind of quantitative methodology called multi-agent simulations based on relatively recent developments in computer science suits well precisely for the analysis of complex social systems and emergent phenomena. It falls within the family of quantitative research methods because simulations generate great amount of measurable and easily replicable data. Yet it does not necessarily rely only upon simple linear relations of given variables. Complex system means that its outcome(s) and/or functioning are not determinable by force of pure logic, deduction, or mathematical inference (Axelrod 1997: 3). Corresponding models contain many autonomous actors, stochastic variables, multiple possible initial states, feedback loops, and highly interdependent features that render usual forms of analysis impossible.

On the one hand, neither simple causal relations between inputs and outputs, nor easily identifiable (in)dependent variables can be usually found in agentbased models or in related complex social systems. On the other hand, these complex nonlinear relations are easily replicable via multi-agent computer simulations (Axelrod 1997: 3). Repeated interactions of autonomous agents often lead to emergent properties at the higher, systemic level of the model concerned. That's also why one can often draw a clear cut distinction between equation-based approaches to scientific modelling and their agent-based counterparts (Parunak et al. 1998):

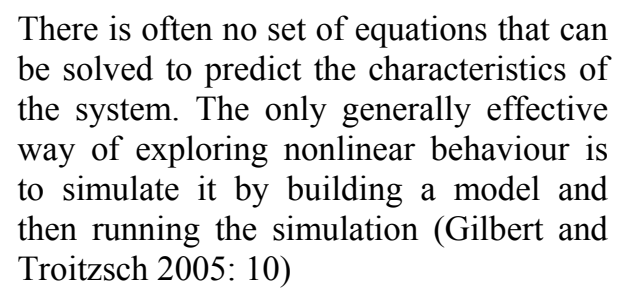

But applying agent-based modelling and multi-agent simulations in social sciences is far from unproblematic. The very nature of a social world asks for special research design and abductive reasoning proposed in this paper offers exactly such a solution.

\section{SIMULATING COMPLEX SOCIAL SYSTEMS}

By repeated runs of the program with variable input parameters computer simulations enable experimentlike research design, which is unseen and unprecedented in social sciences (Gilbert and Troitzsch 2005: 4). When compared with rather rigid computational alternatives, this quantitative research method allows us to get a better grasp of dynamic functioning of any complex system, for example of sovereign states. Moreover, 
feedback loops inclusion facilitates better understanding of the links between micro- and macro-level properties (see Gilbert and Troitzsch 2005: 13). In international politics this corresponds to constitutive relationship of states on the one hand and anarchical environment on the other. Yet the method of multi-agent simulations has besides obvious advantages also several drawbacks.

Precisely because of the character of complex social systems the above mentioned experiments rarely lead to discovery of simple solutions and similarly accurate knowledge as in the case of natural sciences. I'm not saying that there are no feedback loops in problems that natural sciences deal with. But social world composed of interacting human beings actually functions thanks to mind-dependent (i.e. language-dependent, i.e. sociallydependent) non-observables. This sole fact makes social sciences much more interested in exploring constitutive instead of causal relations, and in analyzing agentstructure mutual interdependence.

Logical consistency understood in terms of deductive reasoning would be fine, but unlike in natural sciences, you cannot always take social world for granted. You can rarely deductively infer predictions from general assumption and expect that you will be able to test them under the same conditions whenever you want. One has basically no influence over mindindependent natural phenomena, but the very existence of objects of inquiry in social sciences is at the end necessarily dependent on human (i.e. observer's) action. Under normal atmospheric pressure, $100{ }^{\circ} \mathrm{C}$ will be the boiling point of water regardless of whether we have concepts to describe such a discovery or make any use of it. On the other hand, arguably the last witch in Slovakia was burned in 1741 . There are no witches any more and this is not the case of human caused extinction like that of moa or dodo. People just ceased to act accordingly. Similarly, anarchy among states can favour either cooperative or defective pattern of interactions.

Better knowledge of some complex social system does not necessarily mean greater capability to predict its future development. You don't usually have the opportunity to collect data via real-world experiments and compare them with simulation outcomes in order to calibrate the model (see Tesfatsion 2006: 845).

[T] he best one can do is to test that there is a reasonable likelihood that the observed behaviour of the target could be drawn from the distribution of outputs from the model - which is rather a weak test. (Gilbert and Troitzsch 2005: 212)

For example in natural sciences you can simulate flow of the air past the aircraft's wing, then build the airplane itself, and finally try, if it actually flies. Similarly, you can model avalanche occurrence in a given valley, then arrange avalanche barriers, and see if they work. If real world events do not correspond to simulation results, you can calibrate the input variables and try again.

There is usually no such option in a complex social world. You can model international relations system, but you cannot experiment with the real one, or look for some other example. Your options are rather limited, when your simulation results are different from actual behaviour of the target and given that there is no large $\mathrm{N}$ of comparable real world cases. In fact you can hardly know whether it is your model that is flawed, or simply what you observe in the real world is just an accidental development of the system that usually evolves in a completely different way. Moreover, prediction based on outcomes of simulations is possible only if the model offers very accurate picture of the real world target. Thanks to dynamic and complex character of social world we almost generally lack comprehensive enough knowledge necessary to achieve this level of accuracy (see Gilbert and Troitzsch 2005: 23 \& 18-9 for validation problems of highly abstract models).

Hence if not testable predictions, causality, and accuracy, then we must simply choose other options with greater level of abstraction (Macy and Willer 2002: 146-7; Gilbert and Troitzsch 2005: 26; Simon 1996: 16). But here we come across another peculiar issue in social sciences, namely that of objective knowledge. Observer and observed are often inseparable here.

[T] here are two plausible stories to tell, one from outside about the human part of the natural world and the other from inside a separate social real. One seeks to explain, the other to understand. (Hollis and Smith 1991: 6; emphasis added)

Explanation makes sense only if we accept the key role for causal reasoning, possibility of objective knowledge, and ability to identify (in)dependent variables. All of that is unproblematic with respect to rationalist thinking, but rather difficult to achieve when modelling complex social systems.

However, certain epistemological position that emphasizes subjective understanding of the social world instead of objective causal explanations still remains (Hollis and Smith 1991). In contrast with rationalists, proponents of this approach are often labelled as interpretativists or reflectivists (Keohane 1988). The problem here is that it would be a bit overstretched to include multi-agent simulations among interpretative qualitative methods, although they are often employed by theories focused on constitutive relations as well. Despite all above mentioned problems of multi-agent simulations, choosing 'understanding' (Axelrod 1997; Gilbert and Troitzsch 2005) as the only alternative that remains available after rejecting explanation and prediction is a rather misleading decision. It unmasks little awareness of the debate on epistemological issues, and maybe also still unsettled terminology of the research program dealing with agent-based modelling.

If we disregard those ends of computer simulations that relate to training of some skills (flight simulators) or entertainment (Xbox, PlayStation), we will basically end up with either already rejected prediction of events, or simple urge to better grasp some insufficiently explored phenomena. Thus exploration rather than 
understanding seems to be a better term for what multiagent simulations can achieve. If your goal is to explore functioning of a complex social system, your research design must stay somewhere in between unattainable explanation and inadequate understanding.

What I am trying to say is that similarly as in ontological matters, where agent-based modelling embraced parts of individualist as well as structuralist theories, so also in case of epistemology the multi-agent simulations can take the middle position between explanation and understanding in order to explore how complex systems work. At the same time, this particular computer simulation method cannot be associated with some specific theoretical position or school. Although methodological individualism of classical game theory did in fact facilitate effective application of multi-agent simulations upon complex systems research.

Despite its drawbacks the method concerned enables generation of large amount of replicable, quantifiable data, and thus compensates for lacking experiments within the social world. It helps us explore functioning of complex systems and under specific circumstances even propose their perfection in a desired way. In fact, multi-agent simulations and related agent-based models seem to be the best way how to analyze the macro-level of many social systems such as that of sovereign states. What one has to do now is propose a general framework for a research design that will be adjusted to needs of agent-based modelling of complex social systems.

\section{ABDUCTIVE REASONNING}

Any agent-based enterprise seems to start in a usual deductive manner by constructing a model with help of general assumptions about the composition and ruling principles governing the target. For example, which states interact with each other, how often, and with what possible outcomes? Yet research in the next step does not proceed to compare predictions inferred from these general assumptions with data acquired by observing the real world. Instead, computer simulations generate own data and from them we inductively generalize upon functioning of the modelled target itself. Thus if our model of international relations leads to cooperative behaviour of states, we infer that the real world system favours cooperation too. Agent-based models and multiagent simulations then for some naturally represent the "third way of doing science" besides widely accepted deductive and inductive reasoning (Axelrod 1997: 3-4). Basically, if we find some complex phenomenon such as peace or segregation that needs to be understood better, but cannot because of the missing data or lacking analytical tools, we construct an agent-based model and run the associated multi-agent simulations.

Apart from deductive and inductive reasoning there really is the third option well suited for agent-based modelling. It is called abductive reasoning and it was developed by Charles Peirce, who saw it as the only method truly generating new ideas (see 1994: 2.96 \& 5.145; in early writings he even dubbed it hypothesis, retroduction, or alternatively presumption). Some called it "pragmatic research strategy" and argued that:

abduction should be at the center of our methodological efforts while deduction and induction are important but auxiliary tools. Abduction follows the predicament that (social) science is, or should be, above all a more conscious and systematic version of the way by which humans have learned to solve problems and generate knowledge in their everyday lives. (Friedrichs and Kratochwil 2009: 709-10)

According to Peirce himself, who understood the scientific process in terms of unity of all three types of inferential reasoning, abduction generates new hypothesis, deduction draws predictions, and induction puts them under test (Peirce 1994: 5.171 \& 7.218).

However, abduction raised serious doubts among scholars mostly because of the way how Peirce described it (see e.g. Kapitan 1992; but also Hoffmann 1999). On the one hand, he declared that "abduction is, after all, nothing but guessing" (Peirce 1994: 7.219), and that " $[\mathrm{n}] \mathrm{o}$ reason whatsoever can be given for it, as far as I can discover; and it needs no reason, since it merely offers suggestions." (1994: 5.171) Yet on the other hand, he made sure that abduction "is logical inference, asserting its conclusion only problematically or conjecturally, it is true, but nevertheless having a perfectly definite logical form." (Peirce 1994: 5.188) He defined abduction in the following way:

The surprising fact, $\mathrm{C}$, is observed;

But if A were true, $\mathrm{C}$ would be a matter of course Hence, there is reason to suspect that $\mathrm{A}$ is true.

(1994: 5.189; see also 2.623)

Now there is a problem of what presumption we choose, as well as why we choose it? Relationship between two alternative hypotheses is the most contested issue and Peirce remained rather vague with respect to that. How do we discriminate between $\mathrm{A}$ and $\mathrm{B}$, if both claim to account for C? For him, suggesting hypothesis is a matter of insight and some background knowledge. Understandably, this is not enough to put abduction on a par with deduction and induction. Insight does not prevent us from proposing the most foolish hypotheses. Even if Peirce tried to introduce some rules for coming up with new hypotheses, his attempts were seen as insufficient, if not counterproductive. Objections thus remained. How do we infer $\mathrm{A}$ from $\mathrm{C}$, and why should we prefer it, if there are other available hypotheses?

Yet despite all this scepticism, people do make abductive inferences. You do not have to watch $D r$. House to realize that diagnostics is abductive process par excellence, in which physicians try to find out what is the explanation of patient's problems. Reasoning of William of Baskerville from The Name of the Rose by Umberto Eco is a perfect example of the inference nonreducible to deductive or inductive logic too. People regularly propose hypotheses explaining observed data 
and then proceed to test them after drawing predictions as Peirce demanded. While trying to find out what a good abduction requires, he turned his attention to the problem of good explanation (1994: 5.197). His implicit emphasis on explanatory power later enabled perfection of the concept of abductive inference.

Peirce's abduction is now generally identified with more developed and refined version called inference to the best explanation (Harman 1965; Lipton 2004), which seems to solve the problem of both what hypothesis we draw from available data, as well as why we prefer that particular hypothesis. Of course, Lipton understood inference to the best explanation as a principle of inductive rather than abductive reasoning. But his extremely broad definition of induction actually included all non-demonstrative reasons (2004: 5) as opposed to those deductively inferring true conclusions given true premises. Moreover, he cited Peirce as well as Harman as authors that already analyzed the issue he was concerned with (Lipton 2004: 56-57). What follows is thus a description of how inference to the best explanation updated the idea of abductive reasoning.

To shed some light on problems of description (what hypothesis) and preference (why this hypothesis) as articulated by Kapitan but also Lipton, let's start with "the idea that explanatory considerations are an important guide to [abductive] inference, that we work out what to infer from our evidence by thinking about what would explain that evidence" (Lipton 2004: ix). One can already see the connection with Peirce via emphasis on explanation, and inference of hypothesis from given evidence. For understanding Lipton's theory it is also important to notice the difference between inference and explanation. Take four seasons as an example. After observing winter, spring, summer, and autumn in the Central Europe each year, we use inductive generalization to infer that they will continue to alternate also in the future. Yet this inference does not explain why seasons actually change. In order to do so one needs to know obliquity of the Earth as well as its orbital motion around the Sun. Bearing in mind this difference, Lipton defines abduction (or inference to the best explanation, if you want) in the following way:

we infer the explanations precisely because they would, if true, explain the phenomena. Of course, there is always more than one possible explanation for any phenomenon ... so we cannot infer something simply because it is a possible explanation. It must somehow be the best of competing explanations. ... Given our data and our background beliefs, we infer what would if true, provide the best of the competing explanations we can generate of those data ... Far from explanation only coming on the scene after the inferential work is done, the core idea of Inference to the Best Explanation is that explanatory considerations are a guide to inference. (2004: 56)
Obviously this is a much more developed version of abduction than that offered by Peirce. We infer the best possible explanation given available information (the 'what' question), and then we assume that this inference is true, because it is actually the best available explanation (the 'why' question).

But there still remain some questions especially with respect to the quality of being the best explanation. Lipton tried to make his ideas clear by defining 'best' in terms of the loveliest potential explanation (2004: 61). First, he stressed the so called contrastive explanation as a tool that helps us find the best of available options via comparing alternative causal stories: "To explain why $\mathrm{P}$ rather than $\mathrm{Q}$, we must cite a causal difference between $\mathrm{P}$ and not-Q, consisting of a cause of $\mathrm{P}$ and the absence of corresponding event in the case of non-Q" (Lipton 2004: 42). And second, to be considered 'lovely' an explanation must also demonstrate theoretical elegance, simplicity, and unification (Lipton 2004: 68). Also for Harman (1965: 89), being more plausible, simpler, and able to explain more in a less ad hoc manner were the criteria for seeing one hypothesis better than the other.

However, to what extent is all of that applicable upon agent-based modelling of complex social systems? How to apply the criteria for lovely inference, if exploration rather than explanation is the best term for what multi-agent simulations enable? The next section therefore offers the way how to use abductive reasoning for designing research on complex social systems.

\section{ABDUCTIVE FRAMEWORK FOR RESEARCH ON COMPLEX SOCIAL SYSTEMS}

There exist many established criteria for evaluating relevancy and rigor of scientific theories and associated research in social sciences. But the problem is that most of the scholars dealing with scientific criteria assessing various research designs have adopted rationalist view that promotes hypothetico-deductive model of science with the need of empirical falsifiability of examined theories (see Popper, 2002). Thus for example Walt (1999) besides naturally required originality demands also empirical validity and consistency in terms of deductive logic. Famous book by King, Keohane, and Verba (1994) is very similar in this respect. However, given what I wrote above about the observer-observed relationship, about the possibility of real world experiments, and about causality, it seems that the hypothetico-deductive model of enquiry is not always suitable for social science of complex systems.

Taking the world of natural sciences for granted, people can easily begin in a deductive way by making theoretical assumptions, then draw predictions, and finally falsify or corroborate them against the evidence. Of course, certain social phenomena are more stable than the others, and I am not saying that deductive reasoning is something foreign to social sciences. Yet as far as multi-agent simulations of complex social systems are concerned, the real world (experimental) testing of predictions is hardly achievable, or even expressed as a goal. Given the fact that natural 
phenomena are independent of human mind, but social ones are not, there must be a difference between the ways how social and natural sciences do their jobs. Hypothetico-deductive code of conduct can be at best a bonus, rather than the core of research design here.

Abductive reasoning is much better suited as a framework for agent-based modelling in social sciences and for the related computer simulation methodology. At least as far as artificial intelligence is concerned, this has been noticed by other scholars as well (Josephson and Josephson 1996). We simply cannot take the world of social sciences for granted, and thus we have to start with accurate observation assuring that the unexplored object of our research actually still exists. Given that the phenomenon is present and significant, one can proceed to build a model. Plausible and in the best case scenario also empirically valid assumptions of the agent-based model should together with replicable multi-agent simulations lead to growing of the modelled phenomena. If this is achieved, one can finally conclude that it is reasonable to regard assumptions of the model and their consequences as correct, hence successfully exploring functioning of the complex system. There is no need for, and the possibility of, real world experiments or deductive reasoning so far. Only thereafter we may inductively (from simulation results) or deductively (from model's assumptions) infer some predictions with respect to the real world. Manipulation of particular features of the target, if possible, will then enable us to examine, if these predictions really hold.

For example if we want to explore cooperation among states in anarchic international environment that for realists leads only to war, we can propose a model with few assumptions based on available information. Fundamental units would be states with different rules of behaviour and interacting in the Prisoner's Dilemma with frequency of encounters dependent on their power and distance. There would be a possibility of friendship and enmity as well as various kinds of mistakes. If such a model favours cooperative behaviour of agents then we can draw some conclusions about the real world target, which thus becomes better explored. Moreover, from results received we can make further inferences about the impact of different variables (such as noise, power etc.) upon functioning of our target. One can for instance test simulated impact of noise by improving access to information. This is a common role for international regimes and institutions. Yet we must still bear in mind the difficulty to conduct real world experiments with the complex social systems and also the possibility of multiple alternative outcomes.

With respect to the original definition of abduction by Peirce (1994: 5.189) we can thus propose its reformulation for agent-based modelling:

An unexplored emergent phenomenon of some complex social system is observed and agent-based model of corresponding complex system is then constructed. If multi-agent simulations lead to growing of the emergent phenomena, then there is a reason to suspect that assumptions of the model are correct.

As one can see, we proceed from the given evidence or observations to the formulation of a new hypothesis (model), which in case of successful simulations justifies abductive inference regarding its validity. Yet as with the original definition, modified version of abduction fitted for agent-based modelling needs a more detailed description of the way how we move between premises of the argument, i.e. how we get from the observation to the particular form of the hypothesis. Similarly, more has to be said also about how to discriminate between different available models and what is the place for subsequent empirical testing?

I tried to show above that generally accepted criteria for evaluating research in social sciences based on hypothetico-deductive framework are not very helpful here. Nevertheless, we can still draw some inspiration from them and refine these rules according to the logic of inference to the best explanation. Thus requirements that any good agent-based research design has to meet include real world and scientific significance, (intuitive) plausibility of assumptions, and replicable data.

The first characteristic of every sound agent-based model using multi-agent computer simulations is the significance both with respect to the real world and the scientific community as well. This criterion was not only expressed by King, Keohane, and Verba (1994: $15)$, but it is present in Walt's originality requirement too (1999: 9). Significance means not only that your research (e.g. robustness of peaceful interactions among states) must have a real impact on human life, but it must also enhance the scientific knowledge so that your model is in an important way different from the others.

The second feature of a good agent-based research design is the plausibility of its assumptions. This is also a kind of shortcut for deciding, which of the possible models counts as the best (or loveliest) exploratory tool for a given complex system. Plausibility is achieved theoretically or empirically, but ideally via both ways simultaneously. Necessity to defend different features of the model in both ways limits the number of possible alternatives. For example one can hardly justify actors moving on a playing grid, if you model sovereign states.

However, you can still include many additional features or modify the present ones so that your model will correspond better to the real world target. What to include and what to left behind is an important issue related to the question of model's plausibility (Gilbert Troitzsch, 2005: 19). To get the loveliest solution one has to strike a compromise between maximizing accuracy and simplicity. On the one hand, there are highly complicated models with many details such as territorial extent or the political system. They commonly represent what Lipton called 'likeliness'. By producing an accurate copy of the modelled complex system they try to increase the probability of desired results (emergent phenomena). The problem is that many features of such models have none or only very little impact upon simulation results and could have been 
omitted altogether. Allocation of resources, territorial growth, ethnic composition of the population, all these features are probably redundant if you model the system of states. Notice also that loveliness and likeliness are not disjunctive properties in case of inference to the best explanation (Lipton 2004). Lovely model can be easily also the most likely to produce desired results.

On the other hand, there are extremely abstract models with only very few assumptions. If they are successful in growing the emergent phenomena, they can offer exploratory qualities of unmatched loveliness. And even without contrastive explanation one can still reduce number of assumptions as far as the model intuitively makes sense and resembles the target. But many times, abstraction goes on account of empirical and theoretical plausibility. You can hardly justify round-robin encounters in the model of international relations. Even if it is the simplest possible pattern of interactions, states do not meet each other equally often. Deciding what to include in any agent-based model requires striking a balance between simplicity and accuracy by asking in a Lipton's way 'Why this feature rather than the other?' In order to maximize loveliness of the model in form of elegance, unification, and simplicity (Lipton 2004: 68) one always has to keep in mind overall empirical and theoretical plausibility.

Finally, for every sound research design it should be a matter of fact, that if the model built upon plausible assumptions leads towards successful growing of some phenomenon, then the simulation results must also be readily replicable (see Axelrod 2003). But the problem of replicable data has several aspects besides that of ability to reproduce outcomes by other members of the scientific community (one of the very few examples is Axtell et al., 1996). To prove that results are not artefacts, one must first and foremost be sure that they are not caused by some bug in the source code. Nevertheless, debugging is only the fourth out of six steps of modelling research design according to Gilbert and Troitzsch (2005: 19; see the Figure below).

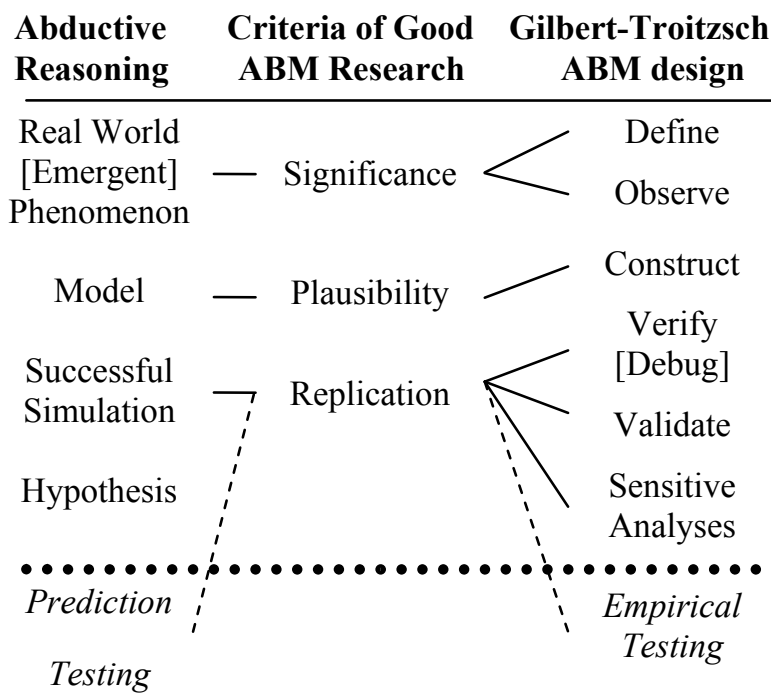

Figure 1: Template for Agent-Based Modelling of Complex Systems.
Debugging is preceded by definition of the puzzle, observation of the target, and construction of the model. The first two stages correspond to the initial step of the abductive inference as defined above and portrayed on the left side of the Figure 1. The fifth stage called validation by Gilbert and Troitzsch aims at securing the match between outcomes of simulations and functioning of the target. Together with the fourth (debugging) and the sixth step (sensitivity analysis) it fits nicely within the part of abductive reasoning trying to evaluate model-target correspondence. These three last steps also find their place within broadly defined replication criterion of a good agent-based research design.

I already said a lot about the problems of acquiring heterogeneous enough data from complex social systems. Predictions are rare. Real world experiments are impossible. And small number of comparable cases together with complicated nature of feedback loops prevents proper statistical analysis. Hence requiring the empirical testing as a part of the replication criterion seems many times unrealistic. Yet Peirce expected that after provisionally accepting the hypothesis abductively inferred from available evidence, research will proceed by way of deduction "to trace out its necessary and probable experiential consequences" (Peirce 1994: 7.203). Similarly, Lipton stressed the possibility of using inference to the best explanation for creating high level theories, and then deducing the lower level hypotheses and predictions in an opposite direction (2004: 63). Not all agent-based models are equally complex and there can actually be some chance for making deductive inferences. But even if deduction becomes unavailable, one can still turn to sensitivity analysis and inductive generalizations.

Considering the best possible scenario in which plausibly constructed model leads to simulation results that accurately mirror targeted phenomena (remember that this correspondence can still be accidental), then the most one can do in case of many models is really only to conduct sensitivity analysis as the penultimate step under the Gilbert-Troitzsch ABM research design. By manipulating input parameters of individual reruns of the program one can try to isolate their impact upon simulation outcomes. Subsequent generalization can suggest modifications of those parts of the target that might change functioning of the modelled complex system in a desired way. Then if the results of these modifications are different from what has been expected, one either has to change assumptions of the model, or run more simulations in order to find undiscovered end states. This is probably the only way how to build a weak but at least to some extent nontrivial data link between the model and the target within the social science modelling of the complex systems.

\section{CONCLUSION}

I came across these epistemological and methodological problems of agent-based modelling when writing my own source code of the agent-based, game-theoretic model of the international relations system. My 
intention was to model the systemic parameters, but I didn't want to be restricted to the current settings in the world affairs. Model was meant to be focused upon systemic properties and not upon some particular and accidental instance that the environment of sovereign states evolved into. Modelling the behaviour of specific states facing each other in some particular situation was definitely not my goal.

The real power of game theory, for both empirical and theoretical purposes, emerges when it is used to generate new findings and understandings rather than to reconstruct individual situations. (Snidal 1985: 27)

Yet with such a research design you inevitable find out that there is a lack of existing data heterogeneous enough for the purposes of your model. You also cannot apply what you discover, because scholars simply do not have the opportunity to experiment with the actual system of states.

Abductive reasoning seemed to be the best solution for these problems. It offered an answer to the question of how to cope with the complex social systems. Inference to the best explanation clarified the purpose of multi-agent simulations as a hypotheses generating method that may or may not be followed by the real world empirical testing. Stated simply, with respect to agent-based modelling of some insufficiently explored social system one has to be content with successful growing of the phenomena concerned via multi-agent simulations. While under other research designs empirical work only starts after formulation of the hypothesis, in case of agent-based models and multiagent simulations of complex social systems this is often the end. Growing some emergent phenomenon out of agents' interactions justifies our decision to see the model as being correct (at least temporarily, until still more lovely model is proposed), because it helped explore the puzzle.

\section{REFERENCES}

Axelrod, R. 1997. The Complexity of Cooperation. Princeton University Press, Princeton.

Axelrod, R. 2003. "Advancing the Art of Simulation in the Social Sciences." Japanese Journal for Management Information System 12, No. 3, 3-16.

Axtell, R.; R. Axelrod; J. Epstein; and M.D. Cohen. 1996. "Aligning Simulation Models: A Case Study and Results.“ Computational and Mathematical Organization Theory 1, No. 2, 123-41.

Bennett, A. and C. Elman. 2007. "Case Study Methods in the International Relations Subfield." Comparative Political Studies 40, No. 2, 170-195.

Friedrichs, J. and F. Kratochwil. 2009. "On Acting and Knowing: How Pragmatism Can Advance International Relations Research and Methodology." International Organization 63, No. 4, 701-31.

Gilbert, N. and K.G. Troitzsch. 2005. Simulation for the Social Scientist. Open University Press, Maidenhead.

Harman, G.H. 1965. "The Inference to the Best Explanation." The Philosophical Review 74, No. 1, 88-95.
Hoffmann, R. 1999. "The independent localisations of interaction and learning in the repeated Prisoner's Dilemma." Theory and Decision 47, No. 1, 57-72.

Hollis, M. and S. Smith. 1991. Explaining and Understanding International Relations. Clarendon Press, Oxford.

Josephson, J.R. and S.G. Josephson. 1996. (Eds.). Abductive Inference. Cambridge University Press, Cambridge.

Kapitan, T. 1992. "Peirce and the Autonomy of Abductive Reasoning." Erkenntnis 37, No. 1, 1-26.

Keohane, R.O. 1986. "Reciprocity in international relations." International Organization 40, No. 1, 1-27.

King, G.; R.O. Keohane; and S. Verba. 1994. Designing Social Inquiry. Princeton University Press, Princeton.

Lipton, P. 2004. Inference to the Best Explanation. $2^{\text {nd }}$ edition, Routledge, Abingdon.

Macy, M.W. and R. Willer. 2002. "From Factors to Actors: Computational Sociology and Agent-Based Modelling." Annual Review of Sociology 28, 143-66.

Parunak, H.v.D.; R. Savit; and R.L. Riolo. 1998. "AgentBased Modelling vs. Equation-Based Modelling: A Case Study and Users' Guide." In Proceedings of Multi-Agent Systems and Agent-Based Simulation. J.S. Sichman et al. (Eds.). Springer Verlag, Berlin, 10-25.

Peirce, C.S. 1994. The Collected Papers of Charles Sanders Peirce. Edited electronic version by J. Deely reproducing Ch. Hartshorne and P. Weiss (Eds. 1931-1935). Vols. IVI; and A.W. Burks (Ed. 1958). Vols. VII-VIII. Harvard University Press, Cambridge.

Popper, K.R. 2002 [1959]. Logic of Scientific Discovery. Routledge, London.

Simon, H.A. 1996 [1969]. The Sciences of the Artificial. 3rd edition, MIT Press, Cambridge.

Snidal, D. 1985. "The Game Theory of International Politics." World Politics 38, No. 1, 25-57.

Tesfatsion, L. 2006. "Agent-based computational economics: a constructive approach to economic theory." In Handbook of Computational Economics: Agent-Based Computational Economics 2. L. Tesfatsion and K.J. Judd (Eds.). North-Holland, Amsterdam \& Oxford, 831-880.

Walt, S. 1999. "Rigor or Rigor Mortis? Rational Choice and Security Studies.” International Security 23, No. 4, 5-48.

\section{AUTHOR BIOGRAPHY}

MATUS HALAS was born in Kosice (Slovakia) in 1984, and graduated with highest honours in Political Science from the Faculty of Arts at the Comenius University in Bratislava. He also received B.A. in European Studies from the very same university. As a postgraduate student of International Relations at the Charles University in Prague he focused on gametheoretic modelling of the system of states. His research interests include agent-based modelling of complex systems, formal theories of international relations, and philosophy of poststructuralism. He published in Perspectives, Mezinarodni vztahy, and Millennium. 